\title{
Poor insight, social avoidance, and better coping predicted prolonged duration of untreated psychosis in schizophrenia
}

Drake RJ, Haley CJ, Akhtar S, et al. Causes and consequences of duration of untreated psychosis in schizophrenia. Br J

Psychiatry 2000 Dec;177:511-5.

QUESTION: In patients with a first admission of schizophrenia, what patterns of symptoms and social functioning predict prolonged duration of untreated psychosis (DUP)?

\section{Design}

Inception cohort with re-interview at 6-12 weeks.

\section{Setting}

3 UK centres (Manchester, Liverpool, and North Nottinghamshire).

\section{Patients}

248 patients who were $16-64$ years of age (median age $27 \mathrm{y}, 70 \% \mathrm{men}$ ) with a first admission of schizophreniform disorder, schizophrenia, schizoaffective disorder, delusional disorder, or psychosis not otherwise specified. Patients were excluded if substance misuse was judged to be the cause of the psychosis.

\section{Assessment of prognostic factors}

DUP was measured from the first onset of delusions and hallucinations. The independent variables were insight, measured by the Positive and Negative Syndrome Scale (PANSS) item G12, social avoidance by the PANSS item G16, and social integration and coping derived from the Social Functioning Scale (SFS). Confounding variables were ethnicity, sex, substance misuse, age at onset, years of education, and all other PANSS items.

\section{Main outcome measures}

Predictors of prolonged DUP and PANSS scores at 6-12 weeks of follow up.

\section{Main results}

DUP ranged from 4-624 weeks (median 12, mean 38 wks). After log transformation, the mean DUP was 14.9 weeks. Prolonged DUP correlated with lack of insight $(\mathrm{p}<0.001)$, poor social integration $(\mathrm{p}=0.004)$, poor volition $(\mathrm{p}=0.02)$, preserved coping skills $(\mathrm{p}=0.04)$, low preoccupation $(\mathrm{p}=0.02)$, and low hostility $(\mathrm{p}=0.03)$ (table). Of patients reassessed with the PANSS at 6-12 weeks (87\%), mean PANSS score decreased by 23.9 points (40\%). Longer DUP predicted less improvement in PANSS $(\mathrm{p}<0.001)$. Greater improvement in PANSS was predicted by better preadmission social integration $(\mathrm{p}=0.046)$. Less improvement was predicted by better preadmission coping $(\mathrm{p}=0.001)$. Initial insight was not associated with change in PANSS $(\mathrm{p}=0.64)$.

\section{Conclusions}

In patients with a first admission of schizophrenia, prolonged duration of untreated psychosis (DUP) was predicted by poor insight, poor social integration, lack of volition, relatively good coping, low preoccupation, and low hostility. Prolonged DUP predicted poorer treatment response at $6-12$ weeks of follow up.

Correlation of prognostic factors with duration of untreated psychosis*

\begin{tabular}{lc} 
Prognostic factor & Correlation \\
\hline Lack of insight & 0.35 \\
\hline Social integration & -0.23 \\
\hline Coping & 0.17 \\
\hline Poor volition & 0.19 \\
\hline Preoccupation & -0.18 \\
\hline Hostility & -0.15 \\
\hline${ }^{*}$ Adjusted for confounding demographic and symptom variables.
\end{tabular}

\section{COMMENTARY}

The prognosis of most medical and surgical illnesses improves if treatment starts early. This correlation has never been shown in psychiatric illness, perhaps because identification and treatment of mental illnesses have, at least in the past, been associated with negative labelling and stigmatisation. More recently, as reported by Drake $e t$ al, many investigators, although not all, ${ }^{1}$ have found a correlation between the DUP and short term illness prognosis: the faster the treatment, the larger the improvement. This correlation may be because the factors that delay the time between having a psychotic symptom and seeking help for it are the same ones that prevent effective treatment response. Or it may be because a lengthy period of untreated psychosis somehow impairs the brain's ability to recuperate. Whatever the case, determining the reasons for delayed treatment is important.

Psychotic symptoms are frightening for the individual and for family and friends. They interrupt school, work, and interpersonal relationships. Allowed to continue unabated, they potentially undermine self confidence, friendships, family cohesion, and academic and vocational success. The study by Drake $e t a l$ is important because it identifies important correlates of delayed treatment: lack of insight, social isolation, and relatively good coping skills. The last factor must be viewed as controversial because other investigators have found that poor premorbid coping correlated with a longer DUP. ${ }^{3}$ The first 2 factors have face validity and important public health relevance. Lack of insight can be addressed by public education. Social isolation can be identified in school. It is time to develop interventions for psychosis that emphasise early detection and address the obstacles to treatment.

Mary V Seeman, MDCM, FRCPC, FACP University of Toronto Toronto, Ontario, Canada

1 Craig TJ, Bromet EJ, Flennig S, et al. Is there an association between duration of untreated psychosis and 24-month clinical outcome in a first-admission series? Am J Psychiatry 2000;157:60-6.

2 Ho BC, Andreasen NC, Flaum M, et al. Untreated initial psychosis: its relation to quality of life and symptom remission in first-episode schizophrenia. Am J Psychiatry 2000;157:80815 .

3 Larsen TK, Moe LC, Vibe-Hansen L, et al. Premorbid functioning versus duration of untreated psychosis in 1 year outcome in first-episode psychosis. Schizophr Res 2000;45:1-9. 